spore formation or to stain them by Gram's method. It is stained faintly and with difficulty by basic stains and is easily decolourised with weak acids and less so with alcohol. Cultivation was unsuccessful both from the blood taken during life and from the blood and organs post mortem. Inoculation with blood taken during life was unsuccessful.

The association of the bacilli with the pigment is supported by their distribution (they are specially numerous in endothelioid and "pigment cells"), by the fact that potassium ferro-cyanide and ammonium sulphide show blue and black granules respectively, which in places are distinctly bacillary and in others bipolar in shape; we have further evidence in the fact that unless the pigment is either fixed or extracted they cannot be demonstrated. Their presence in large numbers in a hyperplastic gland excised very shortly after death and immediateiy hardened excludes the possibility of a post-mortem contamination. Against the hypothesis that they are pathogenic it can be urged that the defensive cells and their generating centres are diseased, have lost their function, and hence the infection may be accidental rather than causative; in answer we have the absence of any other organism in the tissues and the evidences that free and attached endothelioid cells are actively phagocytic to this bacillus. I admit that there is no normal round cell infiltration such as we find in acute infections, but the absence of leucocytes from the blood capable of amoeboid movements should be remembered.

Taking a comparative view of acquired immunity we find that resistance to infective agents is mainly accomplished by phagocytosis and intracellular digestion, and also by other changes, such as the formation of agglutinins and antibodies from leucocytes and every cell in the body in general an important part is also played by the "fixed phagocytes" -connective tissue and endothelial cells. Pathogenic bacteria are also known to protect themselves from antibacterial agents by the formation of impervious capsules or bypertrophy of their sheaths. I may also cite the well-known fact that the tubercle bacillus in a giant cell secretes a fatty envelope which is protective against the ferments of the giant cell, and that the giant cell thus deprived of one means of attack deposits calcium salts, \&c., around or into this envelope, in order to isolate the bacillus and its products. It is possible that pigmentation such as we find in this case is an analogous process. The immanising influence of fats and fatty substances is also of common occurrence. ${ }^{2}$ These facts fit in with the chemical and physical characters of the pigment found in this case. On the little evidence we have at present on one case only we cannot decide whether this bacillus is pathogenic or merely living as an accidental saprophyte, but the facts are significant and I favour the former view. I regard this disease as one in which the first effects fall on the cellular elements of the blood or on their germinal centres, thus depriving the body of its chief defence, which now has to be performed by minor and less perfect means, such as phagocytosis by endothelioid cells and the formation and deposition of impervious substances. In the meantime further investigations into diseases showing leukæ nic changes are necessary. It remains to account for the masses of lymphoid cells. I do not consider them to be wholly comparable to the infective granulomata but rather to be collections of already damaged leucocytes deposited in various places, such as lymph spaces around visceral vessels and subperiosteal spaces.

My thanks are due to Dr. Pope for his permission to carry out and publish my investigations and to Mr. C. J. Bond for his valuable suggestions.

Bibliography-Gustav Lang: Archives Générales de Médecine, November, 1893, and January, February, and Mareh, 1894. Otto Schmidt : U̇ber Einen Fall von Chloroma, Inaug. Dissert., Gottingen, 1895. Koerner: Zeitschrift für Ohrenheilkunde. Band xxix., 1896, p. 96; and Archives of Otologv, New York, 1897, vol. xxvi., p. 289 Paviot et Gallois: Société de Biolngie. Paris, Nov. 14th, 1896; Ibid., Marcin 28th, 1896; Lүon Médicale, 1896, tome lxxxiii., p. 488; Archives Générales de Médecine, Julv, August, and September, 1898. Melville Dunlop: Brit. Med. Jour., May 3rd, 1902. Bvrom Bramwell : Clinical Studies, Edinburgh, 1904-05, vol. iii., p. 29-43. Dock and Warthin Medical News, New York, 1904, vol. xxxv., pp. 971, 1025, 1078, and 1118. C. Sternberg : Beiträge, vol. 11; Pathologische Anatomie Allgemeine Pathologie, Jenit, 1905, vol. xxxvi., pp. 437, 457. T. H Hunt : A Case of Chloroma, Brit. Med. Jour., April 20th, 1907.

1 J. Bordet: Annales de l'Institut Pasteur, Paris, 1897, tome xi., p. 177, Planche V.

2 E. Metchnikoff: Immunity in Infectious Diseases, Paris, 1901, p. 407 .

\section{DISEASES OF THE UPPER RESPIRATORY PASSAGES IN RELATION TO .LIFE ASSURANCE.}

BY W. H. KELSON, M.D. LOND, F.R.C.S. ENG., ASSISTANT SURGEON AND PATHOLOGIST TO THE LONDON THROAT HOSPITAL, GREAT PORTLAND-STIEET, W.

LoOKING through the list of questions to be answered in the medical reports of several life assurance companies taken at random, one is struck by the absence or extreme paucity of reference to the upper respiratory tract, and one is tempted to ask the question,--Are there no serious troubles arising in these parts affecting longevity? or are there no signs nor symptoms to be looked for here which might guide us to the discovery of serious disease in other parts? True there are difficulties obviously in the way of the examination of these regions, more especially the nose, post-nasal space, and larynx, and $I$ am not going to suggest for a moment that any routine examination of these parts should be made in all cases, but, on the other hand, if you will bear with me for a little while, I will endeavour to show that perhaps more complaints than are generally supposed may have their origin here, and that sometimes the very earliest signs of very grave organic disease appear here, and, if recognised, put us at once on our guard in the same way as that in which one is put on the qui vive by unexpectedly coming across albumin in the urine. Also, as regards the nose, the physio$\log y$ of this organ has of recent years had to be rewritten, and its functions have been found to be more nurnerous and important than was formerly supposed and consequently their abrogation by disease more serious.

Let us begin with the larynx, and ask what symptoms are there which should make a laryngoscopical examination of a candidate for life assurance desirable and what physical signs may be detected on so doing, for though the value of any laryngoscopical examination depends largely on the skill and experience of the examiner, yet it may be well to recapitulate briefly some of the principal points to be observed in relation to their value as indicating serious mischief. Speaking generally, we may say that the question of this examination is most likely to arise in young adults of poor physique and any adult about or just beyond middle life. Now the functions of the larynx are respiratory and phonatory, though the existence of the larynx is by no means a necessity for audible intelligible speech or respiration; therefore, the symptoms will be some change in, or interference with, phonation or respiration; and it having been decided to make a laryngoscopical examination, what abnormal appearances may be present to indicate to us the presence of disease which will have a bearing on the prospects of the candidate as regards longevity?

First of all, there may be loss of movement of the vocal cords; that is to say, the outward motion of the cords on getting the patient to take a deep breath, or the inward movement on getting him to phonate, is defective. Now this loss will be due to one of two main causes: either paralysis or mechanical interference. Let us first take paralysis. This may sometimes be due to a toxin, such as in diphtheria or influenza, when it has no speeial significance or may occur as part of a general poisoning such as from lead (two cases of cord paralysis from this cause I have seen) and from arsenic, as reported by Seligmüller and Heymann, but more commonly the paralysis is due to either $(a)$ pressure on the nerve or $(b)$ degeneration of it or its centres. Let us take pressure first. Now the laryngeal muscles, as is common knowledge, are supplied by the recurrent laryngeal nerve, a branch of the vagus, with the sole exception of the cricothyroid, supplied by the superior laryngeal. As to the vexed question whether the ultimate origin of the fibres be the vagus or the spinal accessory, we need not, I think, tronble ourselves this evening, and I may say at once that paralysis from pressure on the vagus above the point of origin of the recurrent laryngeal is very rare but the recurrent itself in the course of development having been caught, as it were, by the aortic arch on the left side and subclavian artery (the representative of the fourth right fœtal arch) on the right, and looping round these structures, is peculiarly exposed to pressure here-e.g., an apical pleurisy or a pericarditis may

I A paper read before the Life Assurance Medical Officers' Associa tion on March 6 th, 1907. 
catch it and paralyse it and so may mediastinal growths or enlarged glands, but more important than these, because very fatal and often latent, is aneurysm of the aortic arch.

Referring to Dr. F. de Havilland Hall's well-known work on "The Medical Examination for Life Assurance" he says : "The records of life assurance offices show that thoracic aneurysms are frequently overlooked. In many instances this omission is almost unavoidable, as even when a patient is anxions to give all the information he can the physical signs are often too ill-defined to enable a diagnosis to be arrived at." This I heartily endorse, at the same time suggesting that if these cases had had their larynges examined perhaps danger signals might have been detected in some of them. In these cases of aortic aneurysm the left nerve, as might be expected, is the one generally affected, but sometimes both are involved; and the cause of this has led to much discussion, some explaining it on the ground of the aneurysmal dilatation having extended into the right subclavian and involving the right recurrent as well as the left; but it has also been shown that pressure on one vagus may, by acting reflexly through the afferent fibres, produce paralysis of motor fibres on both sides.

The onset of paralysis is insidions and peculiar, and with reference to it I must quote the work of Sir Felix Semon, whose investigations in this, as in other matters on laryngology, are of marked and permanent value. Semon's law says : "In organic progressive diseases of the roots and trunks of the spinal accessory, vagus, and recurrent nerves the dilator fibres are affected earlier than the constrictor fibres, or may be even attacked exclusively," and the law holds good in this case. Now the dilator muscle of the larynx or abductor of the cords is the crico-arytenoideus posticus, and it is better to speak of posticus paralysis rather than abductor, because the terms "abductor" and "adductor" are so much alike that confusion is apt to arise. Therefore, if one sees the larynx early it is noticed that the posticus or dilator only is paralysed and the vocal cord lies motionless in the middle line ; in this stage, however, when one cord only is affected, there may be very little, if any, alteration in the breathing or phonation, the outward movement of the cord on the unaffected side during inspiration giving sufficient room. Should, however, both postici be paralysed, both cords lying near the middle line, there is no room to breathe and the danger of suffocation is great. Though neither voice nor breathing may give much warning in this early stage of paralysis of one cord, yet in some cases attacks of difficult breathing and alteration of voice due to spasm of the muscles of the cords occur, the cause being irritation of the nerves. If, however, the condition has passed on to complete paralysis of one cord the voice is weak and more or less hoarse and sometimes with a tendency to falsetto when the patient attempts to speak loudly, but there is no dyspncea; on examining laryngoscopically it will be found that the paralysed cord lies motionless in a position very near to that which it assumes in the dead body and which is known as the cadaveric position. On making the patient phonate it may be seen that the healthy cord comes right across over the middle line and approximates itself to the paralysed one, so helping its weak partner to produce a voice.

Althongh complete left-sided paralysis is the commonest form observed, yet one may get various combinations, such as complete paralysis on one side and partial on the other, the difficulties being phonatory when there is complete paralysis and respiratory when the dilators only are affected. It has been pointed out that the tactile vocal fremitus is different on the two sides of the larynx when one cord only is paralysed, but $I$ do not think much weight can be attached to this. Movement, too, of course, may be communicated to the trachea and larynx by the tugging of the aneurysm especially when the patient's head is held backwards. Also, seeing that the aorta lies close to the anterior surface of the trachea just above its bifarcation and then curves backwards and to the left over the left bronchus, one may find stenosis and stridulous breathing from pressure here, and if the glottis can be well dilated one may sometimes see the pulsation with the laryngoscope in the lower part of the trachea, especially on its left anterior wall.

Leaving now the aorta and aneurysm, let us turn our attention to another point at which the recurrent laryngeal nerves are often pressed upon with resulting paralysis of vocal cords. These nerves, you will remember, passing upwards and backwards behind the subclavian on the right ide and the aorta on the left, reach the groove between the trachea and cesophagus, and continaing their course towards the larynx reach the lower border of the cricoid cartilage where they pass beneath the inferior constrictor muscles of the pharynx, and should they be caught in this region a serious significance must often be attached thereto. Let me illustrate what I mean by a case. In 1897 a man brought his wife, aged 40 years, to me, stating that he was thinking of insuring her life, but wished me just to examine her to see if her various organs were in a sound condition. In answer to my inquiries as to her state of health she stated that she enjoyed very good bealth indeed, but had lately had a slight hoarseness and a little pain in the right ear which she considered to be due to a cold. She had no difficulty in swallowing, but a curious point was that she had recently nursed a sister who had, she stated, died from cancer of the tongue. On making a laryngoscopical examination I found that she had complete paralysis of the right vocal cord, and at the base of the arytenoid on this side there seemed to be some fulness on its osophageal aspect, but nothing could be felt externally, nor was there pain or tenderness here, but I could not help feeling highly suspicious as to the nature of this swelling, thinking that one had in all probability to deal with a malignant growth of the cesophagus in an early stoge. This opinion was confirmed by Dr. H. Lambert Lack, who saw her at my request shortly afterwards. In March, 1899 (about two years later), I saw her again when almost moribund and a few days afterwards was allowed to make a post-mortem examination, when I found a mass of growth involving the recurrent laryngeal nerve on the right side, and this was shown by microscopical examination to be an epithelioma.

Now it would not have been worth while referring to this case but for the fact that this condition is by no means a rare one, and it is a common thing-at any rate, at a throat hospital-to see a patient with commencing malignant disease of the upper part of the cesophagus with very little indeed to show for it except paralysis of a vocal cord. It also occurs in comparatively young persons and $I$ bave seen it myself in a woman aged 28 years. In some goitres also paralysis of the vocal cords may be produced by pressure on the recurrent nerves, but with them external swelling is generally obvious and the examiner is not likely to be taken off his guard.

As regards nerve degenerations, we may find paralysis of the vocal cords in bulbar paralysis, disseminated sclerosis, and syringomyelia, amongst other diseases, but being rarely if ever the earliest symptom of these diseases it has but little importance here from a life assurance point of view, but in tabes dorsalis laryngeal paralysis may be the first sign, and the typical paralysis of tabes is the before-mentioned paralysis of the crico-arytenoideus posticus or abductor of the cord. This may occur on one side or both, but various forms of paralysis may be met with, and the condition may be so marked as to produce a very noticeable alteration in the voice or respiration, or so sljght in effect as to be hardly observable, and the paralysis also may develop very gradually. Besides, irregular or ataxic movements of the cords may occur on the patient attempting to take a deep inspiration or use the voice, which latter may be interrupted and the speech jerky; or there may be the history of laryngeal crises, in which the patient generally feels an irritation or tickling in the throat and gets a violent convulsive cough with noisy respiration and dyspros. The attack is often followed by paralysis of the cords, so that if owing to any such history or to hoarseness or dysproea we examine the larynx and find paralysis locomotor ataxia is one of the diseases to be thought of and confirmatory evidence should be sought for in the ejes, knee-jerks, \&c. In general paralysis of the insane-a very fatal disease-the patient very of ten exhibits more or less marked costicus paralysis, and a case in which laryngeal spasm was the precursor of the disease has been recorded.

Let us now leave paralysis of the recurrent laryngeal nerve and pass on to the conditions in which phonation and respiration are interfered with by mechanical causes in the larynx, fiust, however, remarking that ankylosis of the crico-arytenoid joints may produce appearances and loss of movement very similar to those due to paralysis, but sometimes swelling can be noted round the joint in ankylosis and there is more rigidity of the parts. As regards the intra laryngeal mechanical causes of loss of voice and interference with breathing, they are many, but from a life assurance point of view three only may be regarded as sufficiently common and important to dwell on-viz., (1) malignant 
disease ; (2) tuberculous; and (3) syphilitic lesions. Taking first malignant disease, this affection loses some of its significance owing to the large proportion of cases which occur at over 50 years of age, but a considerable number are met with below 50 , though of late years operative treatment-for such as are dealt with in the early stageshas rendered the prognosis much more hopeful. Fortunately hoarseness is commonly a very early symptom, a usual situation for the growth being one of the true vocal cords; therefore, suspicion being aroused, a laryngoscopical examination should be made, and in proportion as any growth present shows a tendency to infiltrate the parts and interfere with movement by its infiltration so the probability of its being of a serious nature is increased. Removal of a portion of the growth and its examination by a skilled pathologist is in doubtful cases the only way in which the innocence of a growth can be proved, and unfortunately here the objection is constantly raised that the piece removed may not have been deep enough to give a fair sample of the growth itself, and to get a sufficiently large specimen for a decisive opinion is often well-nigh impossible without doing serious damage to the parts In the early stages of intra-laryngeal malignant disease glandular enlargement is improbable.

Laryngeal tuberculosis may be either primary or secondary, though the primary form is undoubtedly rare and opportunities of proving by post-mortem examination that it may exist are rarer still ; yet, on the other hand, there are many cases in which the lung signs are very slight and others in which lung indications seem absent, but subsequent history shows that in all probability the lung affection preceded the laryngeal disease. These cases have considerable import. ance from a life assurance point of view, as they occur in young adults and more often in men than women, and also because the laryngeal symptoms may draw attention to the presence of pulmonary tuberculosis which possibly might be otherwise overlooked. In these cases there is commonly only hoarseness at first with perhaps tickling or pricking in the throat and irritable cough, and sometimes the history of a previous attack of laryngeal catarrh. On examination the first thing as a rule that strikes one is the peculiar pallor of the larynx, and the commonest position for the tuberculous deposit is the inter-arytenoid space where it may either form one or more slight swellings or a distinct tumour jutting out, so as to prevent the vocal cords coming into opposition and so interfering with voice production. The vocal cords and epiglottis are also commonly affected, but one of the points in differential diagnosis between an ordinary catarrh and these more serious tronbles is the less general involvement of the parts in these latter. Thus one cord being swollen and not the other is a suspicious sign; of course, finding tubercle bacilli would clinch the diagnosis, but at this stage the sputa may be very scanty and contain none.

With regard to syphilis, in the secondary stage of this disease hoarseness is common, and I have known this lead to the discovery of the presence of this affection. In the tertiary stage it frequently affects the larynx, and then, as in other parts, simulates malignant and tuberculous disease and may attack any part. Speaking generally, it shows less tendency to warty growth and insidious infiltration than malignant disease and more dusky redness than tubercle. Age, sex, and past history help sometimes, but are apt to prove deceptive. The swelling is often marked and has the special danger here of interfering with respiration at first and also later sometimes by troublesome fibroid contraction.

Let us now pass on to the nose and naso-pharynx. Referring to books on physiology of 20 years ago we find, although referred to in a vague sort of way as affording the natural passage for respiration, yet the nose was accredited with very little beyond that of being the organ for the sense of smell, but owing to the work of Bloch, MacDonald, and others it has been shown to have certain duties in addition, and, if anything, more important-that is, more closely connected with health than olfactory ones. The inhalation of air at an improper temperature, or of too great a degree of dryness, or full of impurities is detrimental to the lower air passages and is apt to be a source of disease, but by scientific experiment it has been shown that however cold or hot within a very considerable range $\left(-7^{\circ} \mathrm{C}\right.$. to $45^{\circ} \mathrm{C}$., MacDonald) external air may be, after passing through the normal rose it will be reduced to approximately th 3 tem rerature of the blood, and at the same time, if dry, it will be saturated with moisture. Impurities are also removed; in fact, some have attributed a bactericidal action to the nasal mucus. These facts being allowed, there can be no doubt that all blockages here, whether due to adenoids, polypi, cartilaginous, bony, or polypoid growths, or consisting of hypartrophied mucous membrane, \&c., by interfering with the proper air-way through the nose, have a very deleterious effect on the respiratory organs below and indirectly damage the general health and, at any rate, predispose to bronchitis, pneumonia, and pulmonary phthisis, which figure so largely in the tables of mortality of insurance offices. Besides, associated with the obstructed nose we get mal-development of the jaws and with them the teeth, so that mastication is interfered with and digestive troubles arise; also associated with congestion and obstructed drainage of mucus from the nose and its accessory cavities are middle-ear catarrh and suppuration. So marked is this in the case of adenoids that the suppuration often ceases like magic when the growths are removed. Besides these, there are other conditions here which bear upon the question of longevity. Let us just take the signs of epistaxis. This occurs commonly enough in children, where it has no special interest for us, but in young adults one must remember the possibility of fibrous polypus and sarcoma, hæmophilia, and the oncoming of typhoid fever and the various forms of anæmia, and it may be an early symptom of splenic leucæmia -and one must not forget that the lungs are often blamed for hæmorrhage, the seat of which is really the nose, the mouth, the pharynz, or the larynx; especially is this the case in instances in which spitting of blood is reported after the night's sleep, that is after the recumbent position has been assumed for some hours. As age advances, however, the significance of epistaxis greatly increases and hospital records show that in cases over middle life in which the hæmorrhage has been so severe as to necessitate them becoming in-patients a very large proportion are suffering from visceral disease of a serious character, the most common being cirrhosis of the liver and contracted granular kidney with urine of low specific gravity and a trace of albumin.

Another trouble which has some importance besides a discharge of blood from the nose is a purulent discharge, and this gathers in weight if associated with headache or pain round the orbit, for suppurative disease of the accessory sinuses and ethmoidal cells is common, and from time to time one sees recorded fatal cases arising from these troubles, and instances of this fatal resu't have been collected by Grünwald and others ; but there is reason to believe that owing to the difficulties present in the way of accurate diagnosis this cause of intracranial disease has been overlooked in a good many cases. Considering the very large percentage of deaths claimed by tuberculous disease, it may be well to note how tubercle affects the nose, and it is very significant how close the nasal cavities are to the meninges, and also their being on the direct highway to the lungs, since tuberculous meningitis and pulmonary tuberculosis are so common. A large number of cases of tubercle in the nose have now been collected, and it may be either primary or secondary, the former being the rarer form and taking generally a poly poid shape, whereas infiltration with ulceration is that usual in the secondary variety. Tubercle bacilli have been found over and over again in adenoids and tonsils, though the latter showed no sign thereof externally except enlargement and frequent association with tuberculous lymphatic glands; probably moderate enlargement, apart from this, need not affect a policy.

Seeing that the back of the mouth, the tongue, and the soft palate, together with the pharynx, are used by both the respiratory and alimentary functions as common ground it would seem that consideration of affections of the upper respiratory tract should include these parts though they are often omitted. As regards the tongue, it should always be examined and no question of inaccessibility can be made here, one great reason being the valuable indications it may give of the candidate's past life, history and habits, such as a tendency to chronic alcoholism, as shown by the furred tongue inclined to tremor with a more or less characteristic odour of the breath. In chronic superficial glossitis, also, we have a complaint both common and important with its various forms or stages, as leucoma, psoriasis of the tongue, and the red glazed smooth form. Its exact etiology may be somewhat obscure, but syphilis, excess in smoking, and alcohol (especially spirits), stand out pre-eminently as contributors to its development, and gout and rheumatism are also believed by Mr. H. T. Butlin to be factors. As regards leucoma and cancer this high authority 
says: "Leucoma is undoubtedly a very strong predisposing cause of cancer"; also, "It may almost be thought that the possibility that carcinoma may be developed in connexion with leucoma has been exaggerated, so much has been said and written of it lately, but we believe that the frequency of the event, so far from having been exaggerated, has been underrated, and that careful records will show a much larger proportion of carcinomas, which have been preceded by leucoma." And it may be said at once that if in these cases of leucoma any ulceration with induration be present we may be pretty certain that the carcinomatous stage has actually been reached. In fact, nowhere is a precancerous stage shown more clearly than in the tongue, and the change going on from leucoma to cancer may be sometimes seen in different parts of the organ at the same time. Leucoma is all the more important because the subjective symptoms are apt to be so very trivial and the pain slight, so that the patient may be unaware of its existence till it is pointed out by the examiner. Ulcers of the tongue also are common and must always be looked on with suspicion, for they are not unfrequently the only indication present of active syphilitic disease, and when this is not present gonty dyspepsia or local irritation will be the most probable cause, but in all cases it is desirable to make quite certain what course the ulcer is going to run before giving a definite opinion. Tuberculous ulceration here or in the pharynx is almost always accompanied by well-marked pulmonary tuberculosis.

When examining the tongue a view can easily be obtained of the palate and one sometimes sees evidence of syphilis here in those apparentily unaware of its presence. Also, by getting the candidate to protrude his tongue and say, "Ah!" we can eliminate the presence of any loss of movement of tongue or palate, such as, combined with laryngeal paralysis, we find in diseases affecting the bulbar nuclei or the surface of the medulla.

In concluding these few and I fear rather disconnected remarks I would submit for your consideration the question as to whether such queries as, "Do you ever suffer from hoarseness or difficult breathing ?" "Can you breathe freely through your nose?" "Have you any mattery discharge from it?" and, "Is your tongue sore?"-might not each or any of them be put to candidates. Also, I should like to get the opinions of those present as to in what circumstances the medical examiner should advise his office to postpone the life until a further examination and conclusive opinion can be obtained. Not so very long ago a running ear was not regarded as having any very great import, but facts have shown this to be a matter requiring serious attention from the view of life assurance, and as our knowledge of rhinology and laryngology increases a pus-discharging nose may be found to have perchance an equal or even greater bearing on a candidate's prospects of longevity than a pus-discharging ear.

Cavendish-place, $\mathrm{W}$

\section{SOME CLINICAL OBSERVATIONS ON MUCO-MEMBRANOUS COLITIS.}

BY J. LIDDHLL, M.D. EDIN.,

PHYSICIAN TO THE HaRROGATE ROYAL Bath HOSPITAL.

DURING the last few years the attention of the profession has been increasingly drawn to the affection called mucomembranous colitis. It is an extremely common malady but one that is liable to be overlooked. Its importance is considerable not only on account of the local symptoms but more because of the secondary effects due to an auto. intoxication. It is the toxic poisoning arising from the condition which gives the real importance to the affection, since this toxin seriously impairs the health and gravely interferes with the well-being and usefulness of a large number of people. The principal objects of this communication are, first, to indicate the clinical manifestations useful in helping: us to recognise the malady when there is no history of the evacuation of mucus from the bowel; and secondly, to describe the method of colon irrigation which has proved so beneficial in the treatment of such cases.

Muco-membranous entero-colitis is a chronic malady affecting the colon but occasionally implicating the lower part of the small intestine. The symptoms may run a continuous course or there may be intermissions followed by exacerba. tions. In respect to the local manifestations of the disease
I shall merely indicate these, as it is unnecessary for me to dwell upon them since they have been well described by various English and continental observers. I shall, however, describe more fully the secondary features, not only on account of their importance to the patient but because they are helpful in enabling us to recognise the disease in obscure cases.

Many of the patients who are classified as neurotics or neurasthenics are sufferers from colitis and there is a causal connexion between their neuroses and the colitis. Indeed, so much is this a fact that most of the obscure cases of colitis can be recognised by the nerve symptoms accompanying the condition, especially when they occur in conjunction with other manifestations which I shall mention. Without further preliminary I shall enumerate the symptoms which usually occur in typical cases of colitis.

\section{SYMPTOMS.}

The patients complain of malaise ; they have occasionally depression of spirits and irritability of temper; often there is sleeplessness or disturbed sleep, loss of memory, impairment of mental and bodily faculties; they are easily moved to tears, and they suffer from cold hands and feet. Though these symptoms may not be peculiar to muco-colitis, yet a surprisingly large number who complain of them are subjects of that affection. Should this group of symptoms or most of them occur in a patient then we ought to inquire into the state of the intestine. The cardinal symptoms referable to the abdomen are constipation, pain, and the evacuation of mucus. The constipation generally is of an obstinate and intractable character and occurs in the majority of cases. Sometimes it alternates with diarrhœa, but this is often spurious. A few cases, however, have habitual diarrhcea or looseness of the bowels. There is usually pain or discomfort in the abdomen and this is often referred to the cæcum, where it may be mistaken for appendicitis. The next most frequent site of pain is the sigmoid flexure, especially in those cases where spasm occurs in the descending colon. Here, as in the crecum, we must be careful to distinguish it from ovarian pain. Pain may also occur in the hepatic and splenic flexures, also in the neighbourhood of the umbilicus. In respect to the evacuation of mucus many colitis patients are not aware that they are passing it from the bowels, either because the quantity is small or more often because it is evacuated in a state unrecognisable by the ordinary observer. If there is no history of the evacuation of mucus and we proceed to examine the patients there are certain features which aid us in detecting these obscure cases of colitis.

The first thing to be noticed in cases of long duration is that the skin is toneless and has a dull yellowish or dirtybrown tint. There are sometimes numerous pigmented patches in the skin, which may be elevated or not. The face wrinkles early and in many cases there is a curve round the angles of the mouth. Many patients are thin through atrophy of the muscles and loss of fat. The circulation is weak and the arterial tension is low. The tongue is often very large and beefy-looking, indented at the edges by the teeth, and in cases of long duration is deeply fissured at the edges and on the dorsum. This is characteristic of the condition. When the tongue is coated there is usually gastric catarrh or dilatation of the stomach The lips are often cracked and in severe cases the buccal mucous membrane is affected with tiny ulcers. On examining the abdominal walls we usually find one of two conditions. Either the walls are thin and retracted, due to loss of fat and atrophy of the muscles; or they are loose and pendulous, producing the so-called "batrachian belly." By palpation and percussion information is obtained as to the state of the colon. The ascending and transverse portions are distended, whilst the descending is often contracted, especially in the neigh bourhood of the sigmoid flexure. This contraction feels like a piece of indiarubber tubing. With deep pressure gurgling or crepitation can be felt chiefly in the cæcum. Auscultation often elicits splashing in the transverse colon when the abdomen is given a slight shake. The kidneys are frequently moveable, the right especially so. The fæces are sometimes pale in colour or pale portions may be seen with ordinary motions. Frequently the motions are attenuated, being of about the size of the little finger; this happens when there is a spasm in the descending colon. Again, they may vary considerably in appearance and consistence, sometimes occurring in small rounded masses or shattered and irregular. Scybala are 